\title{
Avaliação de parâmetros clínicos e hematológicos de eqüinos submetidos a um programa de controle estratégico de Amblyomma cajennense (Fabricius, 1787) (Acari: Ixodidae)
}

\author{
[Evaluation of clinical and hematological parameters of equines submitted to a strategic control program of \\ Amblyomma cajennense (Fabricius, 1787) (Acari: Ixodidae)] \\ A.P. Cunha ${ }^{1}$, A.C.P.P. Bello ${ }^{1}$, R.C. Leite $^{2}$, M.M Melo ${ }^{2}$, G.F. Braz ${ }^{1}$, A.C.C.L. Ribeiro ${ }^{3}$, P.R. Oliveira ${ }^{2}$ \\ ${ }^{1}$ Aluno de pós-graduação - EV-UFMG - Belo Horizonte, MG \\ ${ }^{2}$ Escola de Veterinária - UFMG \\ Caixa Postal 567 \\ 30123-970 - Belo Horizonte, MG \\ ${ }^{3}$ Embrapa Gado de Leite - Juiz de Fora, MG
}

\begin{abstract}
RESUMO
Estudaram-se aspectos clínicos e hematológicos em eqüinos submetidos a um programa de controle estratégico de $A$. cajennense. Os tratamentos carrapaticidas foram realizados a cada sete dias e divididos em dois módulos, o primeiro com início em abril de 2004, e o segundo com início em julho do mesmo ano, utilizando-se a base química piretróide - cipermetrina na concentração de 0,015\%. Além do acompanhamento clínico dos animais, foram realizados hemogramas completos antes e após o programa. As dosagens bioquímicas de bilirrubinas, gama-glutamiltransferase (GGT), aspartato aminotransferase (AST), creatina kinase (CK), proteína total, albumina e globulinas, foram realizadas antes, durante e ao final do programa. Os resultados demonstraram que houve uma melhora no quadro hematológico dos animais após o programa de controle. Os tratamentos carrapaticidas, na forma em foram aplicados, não provocaram alterações desfavoráveis nos parâmetros clínicos e hematológicos dos eqüinos. Tais informações podem ser consideradas na busca de alternativas viáveis e seguras para o controle dessa espécie de carrapato.
\end{abstract}

Palavras-chave: equinos, Amblyomma cajennense, controle, hemograma, bioquímica sérica

\section{ABSTRACT}

Clinical and hematological parameters were studied in equines submitted to a strategic control program of Amblyomma cajennense. The acaricide treatments were carried to each seven days and divided in two batteries, the first one began in April 2004 and the second in July 2004. A pyrethroid chemical base - 0.015\% cypermethrin was used. Clinical examinations of the animals and complete hemograms were carried before and after the control program of the tick. Seric dosages of bilirrubins, gamma-glutamiltransferase (GGT), aspartate aminotransferase (AST), creatin kinase (CK), total protein, albumin, and globulins, were carried before and throughout the experiment. The results showed an improvement in the hematological parameters of the animals after the end of control program. The acaricide treatments did not cause undesirable alterations of the clinical and hematological parameters studied. Such information can be considered as viable and safe alternatives for the control of this tick.

Keywords: equines, Amblyomma cajennense, control, hemogram, serum biochemical, pyrethroid

\section{INTRODUÇÃO}

Os carrapatos são parasitos de grande importância para os animais domésticos e silvestres e para o homem (Fonseca, 1997). Amblyomma cajennense (Fabricius, 1787) (Acari: Ixodidae) pertence à família Ixodidae e subfamília Amblyomminae, sua área de distribuição abrange a América do Sul e Central, Sul da América do Norte e Região do Caribe (Aragão, 1936). É um carrapato trioxeno e que apresenta baixa especificidade parasitária, embora os equídeos sejam os hospedeiros preferenciais (Lopes et al., 1998).

Recebido em 29 de setembro de 2006

Aceito em 17 de janeiro de 2008

E-mail: arildocunha@pop.com.br 
As infestações por A. cajennense ocasionam perdas econômicas importantes, em decorrência da queda de produtividade dos animais e dos gastos com o uso incorreto de produtos carrapaticidas (Prata et al., 1996). Além disso, essa espécie de carrapato é incriminada na transmissão de inúmeros patógenos aos animais e ao homem, destacando-se a febre maculosa, uma importante zoonose (Fonseca, 1997; Leite et al., 1998).

Segundo Pinheiro (1987), no Brasil, o controle do $A$. cajennense tem sido feito empiricamente, com o uso de produtos carrapaticidas encontrados no comércio à base de organofosforados, carbamatos, amidinas e piretróides sintéticos, em concentrações recomendadas para o controle do carrapato dos bovinos Rhipicephalus (Boophilus) microplus (Canestrini, 1887). Pinheiro (1987) e Bittencourt et al. (1989), ao avaliarem diferentes bases piretróides, observaram que o controle de $A$. cajennense exige concentrações mais elevadas de carrapaticida e intervalos estratégicos entre os banhos, verificaram, ainda, que as larvas e ninfas são mais sensíveis aos piretróides que os estádios adultos.

Leite et al. (1997) propuseram o controle de $A$. cajennense em eqüinos, por meio da aplicação sucessiva de tratamentos carrapaticidas, a cada sete a dez dias, nos períodos de predominância de larvas e de ninfas nos animais e no ambiente, com o uso de formulações piretróides puras, na forma de concentrados emulsionáveis, para banhos de aspersão ou imersão.

Os piretróides são compostos químicos que atuam nos canais de íons prolongando a excitação neuronal, mas não são diretamente citotóxicos. Promovem o aumento da permeabilidade da membrana, o que conduz a um bloqueio nas trocas de íons sódio e potássio e levam a uma despolarização intermediada pelo ácido gama-aminobutírico (GABA) e ATPase (Miller, 1988; Ray e Forshaw, 2000).

Os piretróides são divididos em duas subclasses (tipo I e tipo II) em decorrência da estrutura química e da síndrome de intoxicação que podem causar em insetos e, também, em mamíferos. Piretróides tipo I são ésteres do ácido crisantemonocarboxílico e produzem hiperexcitabilidade e tremores. Piretróides tipo II são ésteres do ácido crisantemonodicarboxílico e causam salivação, incoordenação motora, hiperexcitabilidade e reflexo de apreensão. Piretróides tipo II atuam nos canais de íons de sódio e também nos canais de íons de cloro (Ray e Forshaw, 2000; Soderlund et al., 2002).

A cipermetrina é um piretróide tipo II (Wolansky et al., 2006). É uma molécula de terceira geração do grupamento fenoxibenzílico, na qual foi introduzido um grupo ciano no fenoxibenzilester com substituição do átomo de hidrogênio pelo átomo de cloro, tornando-a mais fotoestável e com potencialidade biológica maior que os piretróides de primeira e segunda geração (Fulgêncio e Cordovés, 1997). Os piretróides são metabolizados por oxidação, hidrólise ou conjugação (Miller, 1988), mecanismos que podem variar de acordo com a estrutura química da molécula (Ray, 1991).

Considerando a importância do controle do $A$. cajennense e a escassez de informações de literatura a respeito dos efeitos de tratamentos carrapaticidas sobre os parâmetros fisiológicos de eqüinos e possíveis implicações toxicológicas, objetivou-se estudar os aspectos clínicos e hematológicos em eqüinos submetidos a um programa de controle estratégico baseado na proposta de Leite et al. (1997).

\section{MATERIAL E MÉTODOS}

O estudo foi realizado no município de Palma, Zona da Mata, estado de Minas Gerais. As informações climáticas obtidas no Instituto Nacional de Meteorologia ${ }^{1}$ são relativas à estação meteorológica de Santo Antônio de Pádua, RJ, situada a $20 \mathrm{~km}$ do local do estudo, com altitude de 95m, latitude 21\%32'S e longitude $42^{\circ} 09^{\prime} \mathrm{O}$. Foram utilizados 16 eqüinos adultos (11 machos e cinco fêmeas) sem raça definida (SRD), mantidos em regime extensivo com suplementação mineral. Os animais eram destinados ao trabalho, em uma propriedade de bovinocultura de leite. Os animais foram inspecionados quanto à carga parasitária de carrapatos adultos entre outubro de 2003 a março de 2004. A partir de outubro de 2003 foi implantado um esquema para o controle de

${ }^{1}$ INMET, 2005 - Http://www.inmet.gov.br/htlm/clima. 
helmintos, utilizando-se a base albendazole ${ }^{2}$ por via oral a cada três meses.

Durante os períodos de tratamentos carrapaticidas, foram realizados, a cada 14 dias, exames clínicos nos animais, observando-se a temperatura retal, as freqüências cardíaca e respiratória, o pulso arterial, as mucosas, o tempo de perfusão capilar, auscultação abdominal, turgor cutâneo e linfonodos, de acordo com Speirs (1999).

Os tratamentos carrapaticidas foram divididos em dois módulos de banhos: a primeira realizada nos meses de abril e maio, composta por oito banhos a cada sete dias; a segunda no mês de julho, composta por cinco banhos a cada sete dias, de acordo com a metodologia foi proposta por Leite et al. (1997), e considerou os períodos de picos de larvas e ninfas de $A$. cajennense em vida livre e parasitária para a região Sudeste do Brasil (Oliveira, 1998; Labruna et al., 2002), por serem estes estágios mais sensíveis aos carrapaticidas do que os adultos (Pinheiro, 1987; Bittencourt et al., 1989). Utilizou-se um piretróide sintético - cipermetrina a $0,015 \% \%^{3}$. Cada animal, de acordo com o porte, foi pulverizado com três a cinco litros da emulsão carrapaticida. Os banhos foram realizados no período do dia em que a temperatura e a intensidade solar apresentavam-se mais amenas. O primeiro módulo de tratamento teve a duração de 49 dias entre o primeiro e o último banho, e o segundo durou 28 dias, com um intervalo de 42 dias entre ambos.

Para a realização do hemograma, foram coletadas, de cada animal, duas amostras de sangue, por punção da jugular, utilizando-se agulhas descartáveis $(40 \times 12 \mathrm{~mm})$ e tubos vacuolizados com anticoagulante. A primeira amostra foi coletada no dia zero (dia do primeiro tratamento carrapaticida, antes de os animais serem banhados), a qual foi considerada como controle para comparação com a segunda amostra, que foi coletada 28 dias após o último banho do segundo módulo de tratamentos. Analisaram-se os valores de hemácias, hematócrito, hemoglobina, volume corpuscular médio (VCM), hemoglobina corpuscular media

${ }^{2}$ Valbazen ${ }^{\circledR} 10$ Cobalto - Pfizer - São Paulo, Brasil.

${ }^{3}$ Ec-Tox CE $15 \%{ }^{\circledR}$ - Schering-Plough Saúde Animal - São Paulo, Brasil.
(HCM) e concentração de hemoglobina corpuscular media (CHCM), contagem total e diferencial de leucócitos e presença de linfócitos reativos (Verçosa Júnior et al., 2006). As amostras foram processadas pelo princípio de fotometria no equipamento eletrônico $\mathrm{ABC} \mathrm{Vet}^{4}$ (Godoy et al., 2007).

Para as análises da bioquímica sérica, foram realizadas seis coletas de sangue, sendo três para cada módulo de tratamentos carrapaticidas: para o primeiro módulo foram coletadas amostras no dia zero (antes do primeiro tratamento carrapaticida), 15 e 43, e para a segunda no dia zero, 15 e 57 . O procedimento para obtenção das amostras foi semelhante ao descrito para $o$ hemograma, com a exceção de que foram utilizados tubos vacumtainer sem anticoagulante para a obtenção das alíquotas de soro, as quais foram mantidas a $-20^{\circ} \mathrm{C}$, e posteriormente analisadas para os seguintes parâmetros: bilirrubinas total, direta e indireta, gamaglutamiltransferase (GGT), aspartato aminotransferase (AST), creatina Kinase (CK), proteína total, albumina e globulinas (Viana et al., 2007). No cronograma de coletas das amostras, foi considerado o tempo de meia-vida sérica das enzimas analisadas, conforme Kaneko et al. (1997).

As amostras foram processadas utilizando-se kits diagnósticos (Biosystem) baseados no princípio de fotocolorimetria e a leitura efetuada em aparelho espectrofotômetro ${ }^{5}$.

Os resultados do eritrograma e do leucograma foram submetidos ao teste t pareado e os dados da bioquímica sérica ao teste de Tukey, com grau de confiança de 5\% (Sampaio, 2002).

\section{RESULTADOS}

Ao longo do experimento, os parâmetros observados no exame clínico mantiveram-se sempre dentro dos limites de referência para a espécie eqüina, segundo Speirs (1999).

Os valores médios obtidos nos eritrogramas dos eqüinos tratados estão expressos na Tab. 1. Os resultados da contagem de hemácias, do

\footnotetext{
${ }^{4}$ Automated Blood Counter/Animal Counter ${ }^{\circledR}-\mathrm{ABX}$

Diagnostics - São Paulo, Brasil.

${ }^{5}$ Cobas Mira Plus ${ }^{\circledR}$ - Roche - São Paulo, Brasil.
} 


\section{Cunha et al.}

hematócrito e da hemoglobina foram superiores após os tratamentos carrapaticidas em relação à coleta-controle $(\mathrm{P}<0,05)$, para os parâmetros de VCM, HCM e CHCM não houve variação significativa entre as coletas.
Com relação aos resultados dos leucogramas dos eqüinos (leucócitos, bastonetes, segmentados, linfócitos, eosinófilos, monócitos, basófilos e linfócitos reativos), não houve alterações significativas entre as amostras coletadas antes e após os banhos carrapaticidas (Tab. 2).

Tabela 1. Valores médios e limites inferiores e superiores do eritrograma de 16 eqüinos adultos submetidos a um programa de controle estratégico de Amblyomma cajennense - Palma, MG, 2005

\begin{tabular}{|c|c|c|c|}
\hline Eritograma & $\begin{array}{l}\text { Pré-tratamento } \\
\text { (controle) }\end{array}$ & Pós-tratamento & Referência $^{1}$ \\
\hline Hemácias $\left(\times 10^{6} / \mu \mathrm{l}\right)$ & $\begin{array}{c}6,65 \mathrm{a} \\
(5,07-8,49)\end{array}$ & $\begin{array}{c}7,96 \mathrm{~b} \\
(5,61-11,01)\end{array}$ & $6,8-14,43$ \\
\hline Hematócrito (\%) & $\begin{array}{c}29,19 \mathrm{a} \\
(24,00-34,70)\end{array}$ & $\begin{array}{c}36,23 b \\
(28,30-46,80)\end{array}$ & $32-53$ \\
\hline Hemoglobina (g/dl) & $\begin{array}{c}9,66 \mathrm{a} \\
(8,60-11,60)\end{array}$ & $\begin{array}{c}11,93 b \\
(9,90-15,20)\end{array}$ & $11,0-19,0$ \\
\hline VCM (fl) & $\begin{array}{c}43,15 \mathrm{a} \\
(38,25-49,59)\end{array}$ & $\begin{array}{c}46,09 \mathrm{a} \\
(41,04-55,02)\end{array}$ & $37-58$ \\
\hline $\mathrm{HCM}(\mathrm{pg})$ & $\begin{array}{c}14,37 a \\
(12,65-16,53)\end{array}$ & $\begin{array}{c}15,21 \mathrm{a} \\
(13,54-18,34)\end{array}$ & $12,3-19,7$ \\
\hline $\mathrm{CHCM}(\mathrm{g} / \mathrm{dl})$ & $\begin{array}{c}33,29 a \\
(33,08-33,46) \\
\end{array}$ & $\begin{array}{c}32,98 \mathrm{a} \\
(32,20-33,92) \\
\end{array}$ & $31,0-38,6$ \\
\hline
\end{tabular}

Valores seguidos por letras distintas na linha diferem entre si pelo teste t (5\% de significância).

${ }^{1}$ Limites de referência para eqüinos segundo Jain (1986).

Tabela 2. Valores médios e limites inferiores e superiores do leucograma de 16 eqüinos adultos submetidos a um programa de controle estratégico de Amblyomma cajennense - Palma, MG, 2005

\begin{tabular}{|c|c|c|c|}
\hline Leucograma & $\begin{array}{l}\text { Pré-tratamento } \\
\text { (controle) }\end{array}$ & Pós-tratamento & Referência \\
\hline Leucócitos $\left(\mathrm{x} 10^{3} / \mu \mathrm{l}\right)$ & $\begin{array}{c}10,23 a \\
(8,10-14,70)\end{array}$ & $\begin{array}{c}11,67 \mathrm{a} \\
(7,4-15,10)\end{array}$ & $5,4-14,3^{1}$ \\
\hline Bastonetes $\left(\mathrm{x} 10^{3} / \mu \mathrm{l}\right)$ & $0 \mathrm{a}$ & $\begin{array}{c}0,02 \mathrm{a} \\
(0-0,24)\end{array}$ & $0-0,1^{1}$ \\
\hline Segmentados $\left(\times 10^{3} / \mu \mathrm{l}\right)$ & $\begin{array}{c}6,20 \mathrm{a} \\
(3,91-9,83)\end{array}$ & $\begin{array}{c}6,12 \mathrm{a} \\
(4,23-8,15)\end{array}$ & $2,3-8,5^{1}$ \\
\hline Linfócitos $\left(\mathrm{x} 10^{3} / \mu \mathrm{l}\right)$ & $\begin{array}{c}3,65 \mathrm{a} \\
(1,83-5,00)\end{array}$ & $\begin{array}{c}4,94 \mathrm{a} \\
(2,59-7,36)\end{array}$ & $1,5-7,7^{1}$ \\
\hline Eosinófilos $\left(\mathrm{x} 10^{3} / \mu \mathrm{l}\right)$ & $\begin{array}{c}0,20 \mathrm{a} \\
0-1,75\end{array}$ & $\begin{array}{c}0,35 \mathrm{a} \\
(0,10-0,64)\end{array}$ & $0-1^{1}$ \\
\hline Monócitos $\left(\mathrm{x} 10^{3} / \mu \mathrm{l}\right)$ & $\begin{array}{c}0,17 \mathrm{a} \\
(0,08-0,28)\end{array}$ & $\begin{array}{c}0,25 \mathrm{a} \\
(0,09-0,49)\end{array}$ & $0-0,1^{1}$ \\
\hline Basófilos $\left(\times 10^{3} / \mu \mathrm{l}\right)$ & 0 & 0 & $0-0,3^{2}$ \\
\hline Linfócitos reativos $\left(\mathrm{x} 10^{3} / \mu \mathrm{l}\right)$ & 0 & 0 & $0-2^{2}$ \\
\hline
\end{tabular}

Valores seguidos por letras iguais na linha não diferem entre si pelo teste t (5\% de significância).

${ }^{1}$ Limites de referência para eqüinos segundo Jain (1986).

${ }^{2}$ Limites de referência para eqüinos segundo Eades e Bounous (1997). 
$\mathrm{Na}$ Tab. 3 encontram-se os valores médios da bioquímica sérica dos eqüinos (bilirrubina total, direta e indireta, GGT, AST, CK, proteína total, albumina, globulinas e relação albumina:globulinas). Destacando-se variações significativas apenas para os parâmetros de bilirrubina indireta e GGT $(\mathrm{P}<0,05)$.

Tabela 3. Valores médios e limites inferiores e superiores da bioquímica sérica de eqüinos adultos submetidos a um programa de controle estratégico de Amblyomma cajennense - Palma, MG, 2005

\begin{tabular}{|c|c|c|c|c|c|c|c|}
\hline \multirow{3}{*}{$\begin{array}{l}\text { Bioquímica } \\
\text { sérica }\end{array}$} & \multicolumn{6}{|c|}{ Tratamento $^{1}$} & \multirow{3}{*}{ Referência } \\
\hline & \multicolumn{3}{|c|}{$1^{\mathrm{a}}$ módulo de tratamento ${ }^{1}$} & \multicolumn{3}{|c|}{$2^{\mathrm{a}}$ módulo de tratamento ${ }^{2}$} & \\
\hline & Dia zero & Dia 15 & Dia 43 & Dia zero & Dia 15 & Dia 57 & \\
\hline $\begin{array}{l}\text { B. total } \\
(\mathrm{mg} / \mathrm{dl})\end{array}$ & $\begin{array}{c}0,97 \mathrm{a} \\
(0,63-1,16)\end{array}$ & $\begin{array}{c}1,01 \mathrm{a} \\
(0,76-1,71)\end{array}$ & $\begin{array}{c}1,06 a \\
(0,74-1,83)\end{array}$ & $\begin{array}{c}0,98 a \\
(0,66-1,52)\end{array}$ & $\begin{array}{c}1,29 a \\
(0,83-1,74)\end{array}$ & $\begin{array}{c}1,11 \mathrm{a} \\
(1,03-1,24)\end{array}$ & $1-2^{2}$ \\
\hline $\begin{array}{l}\text { B. direta } \\
(\mathrm{mg} / \mathrm{dl})\end{array}$ & $\begin{array}{c}0,31 \mathrm{a} \\
(0,04-0,54)\end{array}$ & $\begin{array}{c}0,41 \mathrm{a} \\
(0,29-0,59)\end{array}$ & $\begin{array}{c}0,38 \mathrm{a} \\
(0,27-0,58)\end{array}$ & $\begin{array}{c}0,28 \mathrm{a} \\
(0,19-0,49)\end{array}$ & $\begin{array}{c}0,28 \mathrm{a} \\
(0,18-0,44)\end{array}$ & $\begin{array}{c}0,27 \mathrm{a} \\
(0,18-0,32)\end{array}$ & $0-0,4^{2}$ \\
\hline $\begin{array}{l}\text { B. indireta } \\
(\mathrm{mg} / \mathrm{dl})\end{array}$ & $\begin{array}{c}0,64 \mathrm{a} \\
(0,39-0,84)\end{array}$ & $\begin{array}{c}0,67 \mathrm{ab} \\
(0,42-0,96)\end{array}$ & $\begin{array}{c}0,68 \mathrm{ab} \\
(0,38-1,25)\end{array}$ & $\begin{array}{c}0,69 \mathrm{ab} \\
(0,41-1,04)\end{array}$ & $\begin{array}{c}1,01 \mathrm{~b} \\
0,65-1,42)\end{array}$ & $\begin{array}{c}0,84 \mathrm{ab} \\
(0,75-0,98)\end{array}$ & $0,2-2^{2}$ \\
\hline GGT (U/1) & $\begin{array}{c}19,44 a \\
(14-28)\end{array}$ & $\begin{array}{c}27,69 \mathrm{~b} \\
(11-35)\end{array}$ & $\begin{array}{c}18,94 a \\
(13-36)\end{array}$ & $\begin{array}{c}17,94 a \\
(11-31)\end{array}$ & $\begin{array}{c}19,38 \mathrm{a} \\
(12-36)\end{array}$ & $\begin{array}{c}14,57 a \\
(8-32)\end{array}$ & $6-32^{3}$ \\
\hline $\operatorname{AST}(\mathrm{U} / 1)$ & $\begin{array}{c}348,62 \mathrm{a} \\
(186-446)\end{array}$ & $\begin{array}{c}371,31 \mathrm{a} \\
(195-624)\end{array}$ & $\begin{array}{c}376,62 \mathrm{a} \\
(295-501)\end{array}$ & $\begin{array}{c}349,12 a \\
(257-461)\end{array}$ & $\begin{array}{c}378,69 a \\
(262-478)\end{array}$ & $\begin{array}{c}343,86 a \\
(283-413)\end{array}$ & $160-412^{3}$ \\
\hline $\mathrm{CK}(\mathrm{U} / \mathrm{l})$ & $\begin{array}{c}319,12 \mathrm{a} \\
(185-725)\end{array}$ & $\begin{array}{c}359,69 a \\
(185-725)\end{array}$ & $\begin{array}{c}390,31 a \\
(245-726)\end{array}$ & $\begin{array}{c}506,56 a \\
(166-1136)\end{array}$ & $\begin{array}{c}420,94 a \\
(248-792)\end{array}$ & $\begin{array}{c}436,71 \mathrm{a} \\
(289-898)\end{array}$ & $60-330^{3}$ \\
\hline P. total (g/dl) & $\begin{array}{c}7,29 a \\
(6,13-8,02)\end{array}$ & $\begin{array}{c}7,23 a \\
(6,14-8,09)\end{array}$ & $\begin{array}{c}7,06 a \\
(6,36-7,76)\end{array}$ & $\begin{array}{c}6,92 \mathrm{a} \\
(6,25-7,80)\end{array}$ & $\begin{array}{c}7,72 \mathrm{a} \\
(5,78-8,21)\end{array}$ & $\begin{array}{c}6,69 a \\
(6,16-7,32)\end{array}$ & $5,6-7,9^{2}$ \\
\hline $\begin{array}{l}\text { Albumina } \\
\text { (g/dl) }\end{array}$ & $\begin{array}{c}2,28 \mathrm{a} \\
(1,80-2,70)\end{array}$ & $\begin{array}{c}2,32 \mathrm{a} \\
(2,00-2,60)\end{array}$ & $\begin{array}{c}2,38 \mathrm{a} \\
(2,00-2,60)\end{array}$ & $\begin{array}{c}2,27 \mathrm{a} \\
(1,90-2,90)\end{array}$ & $\begin{array}{c}2,57 \mathrm{a} \\
(2,30-3,00)\end{array}$ & $\begin{array}{c}2,23 \mathrm{a} \\
(1,90-2,60)\end{array}$ & $2,6-3,7^{2}$ \\
\hline $\begin{array}{l}\text { Globulinas } \\
(\mathrm{g} / \mathrm{dl})\end{array}$ & $\begin{array}{c}5,02 \mathrm{a} \\
(3,83-5,62)\end{array}$ & $\begin{array}{c}4,85 \mathrm{a} \\
(3,84-5,89)\end{array}$ & $\begin{array}{c}4,68 \mathrm{a} \\
(3,86-5,76)\end{array}$ & $\begin{array}{c}4,65 a \\
(3,80-5,60)\end{array}$ & $\begin{array}{c}5,15 \mathrm{a} \\
(3,18-6,37)\end{array}$ & $\begin{array}{c}4,46 a \\
(3,86-5,02)\end{array}$ & $2,62-4,04^{2}$ \\
\hline $\begin{array}{l}\text { Relação A:G } \\
(\mathrm{g} / \mathrm{dl})\end{array}$ & $\begin{array}{c}0,46 \mathrm{a} \\
(0,32-0,60)\end{array}$ & $\begin{array}{c}0,48 \mathrm{a} \\
(0,36-0,62)\end{array}$ & $\begin{array}{c}0,51 \mathrm{a} \\
(0,34-0,64)\end{array}$ & $\begin{array}{c}0,50 \mathrm{a} \\
(0,37-0,74)\end{array}$ & $\begin{array}{c}0,51 \mathrm{a} \\
(0,39-0,81)\end{array}$ & $\begin{array}{c}0,50 \mathrm{a} \\
(0,39-0,60)\end{array}$ & $0,62-1,46^{2}$ \\
\hline
\end{tabular}

${ }^{1}$ Banho carrapaticida com intervalo de sete dias: $1^{\circ}$ módulo - oito banhos, nos meses de abril e maio; $2^{\circ}$ módulo cinco banhos, no mes de julho.

Valores seguidos por letras distintas na linha diferem entre si pelo teste de Tukey ( $5 \%$ de significância).

${ }^{2}$ Limites de referência para eqüinos segundo Kaneko et al. (1997).

${ }^{3}$ Limites de referência para eqüinos segundo Eades e Bounous (1997).

\section{DISCUSSÃO}

Durante o programa de controle estratégico de $A$. cajennense os eqüinos não apresentaram quaisquer alterações comportamentais ou fisiológicas que pudessem ser detectadas ao exame clínico, realizado conforme Speirs, 1999. Pinheiro (1987), utilizando concentrações elevadas de diferentes piretróides em eqüinos, não verificou alterações aparentes. Labruna et al. (2004), ao utilizarem a alfametrina na concentração de $0,01 \%$ em éguas, a cada sete dias ininterruptamente, durante sete meses, não observaram reações adversas após os banhos, sabendo-se que tal concentração foi duas vezes maior que a concentração usualmente recomendada para o carrapato bovino $R$. (B.) microplus. A maioria das toxinas não lesa preferencialmente um tecido, um órgão ou um sistema orgânico isolado, mas freqüentemente acomete diversos órgãos ou sistemas corpóreos ao mesmo tempo (Schmitz, 2000). Segundo Valentine (1990), desordens neurológicas e musculares são sinais clínicos característicos em intoxicações por piretróides. Em algumas situações, entretanto, os animais podem morrer sem apresentar sinais específicos de intoxicação (Galey, 1992).

O contato cutâneo e a ingestão de piretróides podem causar parestesia e irritação gastrintestinal respectivamente. No entanto, a absorção lenta pela pele raramente causa intoxicações sistêmicas (Ray e Forshaw, 2000). Fulgêncio e Cordovés (1997) citam alguns aspectos importantes dos piretróides quando comparados aos inseticidas organofosforados e clorados, tais como: são pouco absorvidos pela pele do hospedeiro, deixam baixo resíduo nos animais utilizados para consumo humano e, além disso, apresentam uma meia-vida curta.

Com relação ao eritrograma dos eqüinos (Tab. 1), os exames realizados após o esquema de controle do carrapato apresentaram valores dentro dos limites de padrão para a espécie 
eqüina (Jain, 1986; Eades e Bounous, 1997). Os valores de hemácias, hematócrito e hemoglobina, entretanto mostraram-se significativamente aumentados em relação à amostragem coletada no dia zero do experimento, o que demonstra a ação do programa de controle em diminuir a infestação por carrapatos, que são grandes espoliadores sangüíneos. Além disso, na primeira amostragem (dia zero) os valores médios de hemácias, hematócrito e hemoglobina apresentaram-se abaixo dos índices referenciados na literatura para os eqüinos, e caracterizando uma anemia normocítica normocrômica.

Serra-Freire (1984) verificou redução dos índices de hemácias, hematócrito, hemoglobina, CHCM e leucócitos, e aumento do VCM e HCM, em bovinos natural e experimentalmente infestados por adultos $A$. cajennense, e relatou que um dos animais, que não morreram pelo parasitismo, necessitou de mais de sete meses para recuperar os valores hematológicos verificados antes da infestação. A presença de helmintos pode colaborar para que os efeitos da infestação pelos carrapatos tornem-se mais severos (Serra-Freire, 1984). Labruna et al. (2002) observaram uma correlação negativa entre o hematócrito e o parasitismo por Dermacentor nitens (Neumann, 1897), em que os maiores picos deste carrapato coincidiam com os menores índices de hematócrito.

Quanto ao leucograma dos eqüinos, não foi observada nenhuma alteração significativa entre as avaliações realizadas antes e após os módulos de tratamentos carrapaticidas (Tab. 2). Nas duas amostragens, os valores mostraram-se dentro dos limites fisiológicos para a espécie eqüina (Eades e Bounous, 1997). Estes achados aliados aos resultados do eritrograma demonstram que os tratamentos carrapaticidas não influenciaram negativamente nos valores do hemograma dos eqüinos.

O perfil da bioquímica sérica dos eqüinos mostrou-se estável durante o programa de controle estratégico de $A$. cajennense. Os valores médios de bilirrubina total e direta, AST e proteína total não apresentaram variações significativas entre as amostragens e nem discrepâncias em relação aos padrões fisiológicos, segundo Kaneko et al. (1997) e Eades e Bounous (1997).
Apesar de se verificarem alterações significativas para bilirrubina indireta e GGT $(\mathrm{P}<0,05)$, tais variações se mantiveram compatíveis com os limites fisiológicos para a espécie (Kaneko et al., 1997; Eades e Bounous, 1997).

Embora os índices de CK tenham se mostrado ligeiramente elevados a partir da segunda amostragem, quando comparados com os valores encontrados na literatura, não houve diferença estatística entre os valores médios das diferentes amostragens $(\mathrm{P}>0,05)$. Segundo Eades e Bounous (1997), os valores de CK devem ser analisados em conjunto com os valores de AST a fim de se determinar a causa, ou não, de distúrbios. De acordo com Barton et al. (2000), basicamente todas as células do organismo contêm a enzima AST, mas as células hepáticas e musculares contêm maiores concentrações. Dessa forma, o valor da AST tem maior significado quando analisado em conjunto com enzimas especificas para o tecido muscular, que no caso é a CK. Portanto, como ocorreu no presente estudo, se somente a CK ou se ambas estiverem aumentadas, é mais provável que a causa tenha origem muscular, caso contrário, pode haver envolvimento hepático. Segundo Silveira (1988), em animais de grande porte, os níveis séricos significantes de CK estão acima de 700U/1. Kramer e Hoffmann (1997) também destacam que somente grandes aumentos dos níveis séricos de CK têm significado clínico, pois até mesmo pequenas contusões, injeções intramusculares ou exercícios físicos produzem aumentos detectáveis de CK, o que pode estar relacionado ao discreto aumento dessas enzimas neste estudo, em que os animais eram submetidos constantemente a exercícios que demandavam grande atividade muscular.

Os valores médios de albumina mostraram um discreto decréscimo, acompanhado de um ligeiro aumento das globulinas e, com conseqüente, discreto declínio da relação albumina:globulinas, com base nos valores citados por Kaneko et al. (1997). Esses valores, porém, se apresentaram estáveis nas diferentes amostragens ( $\mathrm{P}>0,05)$, indicando que não houve influência dos tratamentos na discreta disproteinemia. Os níveis das proteínas séricas podem variar devido a fatores fisiológicos não associados a doenças, tais como, idade, sexo, hormônios, prenhez, lactação, nutrição, estresse e alterações hidroeletrolíticas (Jain, 1986; Kaneko et 
al.,1997). Assim, as variações da bioquímica sérica, observadas neste estudo, não se caracterizaram como decorrentes dos banhos carrapaticidas.

Ressalta-se que os banhos carrapaticidas foram realizados com a concentração recomendada para o controle de $B$. microplus em bovinos $(0,015 \%)$, pois a proposta de controle de $A$. cajennense descrita por Leite et al. (1997) preconiza atingir as fases larval e ninfal, estágios do ciclo de vida deste carrapato mais susceptíveis à ação de carrapaticidas piretróides. Sabe-se que os estádios adultos de $A$. cajennense demandam 1,8 vezes a concentração carrapaticida requerida para combater o B. microplus (Pinheiro, 1987). Diante da possibilidade de se utilizarem bases químicas e concentrações menos tóxicas em períodos determinados, torna-se racional que programas de controle de carrapatos considerem o bem-estar animal, a saúde pública e a preservação ambiental.

Os resultados obtidos são indiretos, uma vez que não foram investigados a presença e os níveis de cipermetrina no organismo dos animais, mas reúnem informações importantes quanto ao uso dessa base química em programas estratégicos e intensivos para o controle do A. cajennense em eqüinos.

\section{AGRADECIMENTOS}

Ao Conselho Nacional de Desenvolvimento Científico e Tecnológico - $\mathrm{CNPq}$, pelo apoio financeiro.

\section{REFERÊNCIAS BIBLIOGRÁFICAS}

ARAGÃO, H.B. Ixodidas brasileiros e de alguns países limítrofes. Mem. Inst. Oswaldo Cruz, v.31, p.759-843, 1936.

BARTON, M.H.; MORRIS, D.D. Doenças do fígado. In: REED, S.M.; BAYLY, W.M. (Ed). Medicina interna eqüina, Rio de Janeiro: Guanabara Koogan, 2000. p.608-634.

BITTENCOURT, V.R.E.; MASSARD， C.L.; GRISI, L. Atividade in vitro de alguns piretróides sintéticos no carrapato Amblyomma cajennense (Fabricius, 1787). Pesq. Agropecu. Bras., v.24, p.1193-1199, 1989.
EADES, S.C.; BOUNOUS, D.I. (Eds). Laboratory profiles of equine diseases. Georgia: Mosby-Year Book, 1997. 304p.

FONSECA, A.H. Doenças transmitidas ao homem e animais por carrapatos que parasitam eqüinos. In: SIMPÓSIO SOBRE CONTROLE DE PARASITOS, 2., 1997, Colina. Anais... Campinas: CGE, 1997. p.1-8.

FULGÊNCIO, A.M.; CORDOVÉS, C.O. Quimismo e uso de piretróides. Hora Vet., v.17, p.33-40, 1997.

GALEY, D.F. Toxicology: diagnostic toxicology. In: ROBINSON, N.E. Current therapy in equine medicine. Philadelphia: Saunders, 1992. v.3, cap.8, p.337-380.

JAIN, N.C. The horse: normal hematology with comments on response to disease. In:

Schalm's veterinary hematology. 4.ed. Philadelphia: Lea e Febiger, 1986. p.140-177.

GODOY, R.F.; SANTANA, A.E.; CAMPOS FILHO, E. et al. Estudo eritroleucométrico e proteinograma sérico do sangue do cordão umbilical e jugular de eqüinos ao nascimento e de suas respectivas mães. Arq. Bras. Med. Vet. Zootec., v.59, p.1376-1381, 2007.

KANEKO, J.J.; HARVEY, J.W.; BRUSS, M.L. (Eds). Clinical biochemistry of domestic animals. California: Academic, 1997. 932p.

KRAMER, J.W.; HOFFMANN, W.E. Clinical enzymology.In: KANEKO, J.J.; HARVEY, J.W.; BRUSS, M.L.(Eds). Clinical biochemistry of domestic animals. 5.ed. California: Academic, 1997. p.303-326.

LABRUNA, M.B.; KASAI, N.; FERREIRA, F. et al. Seasonal dynamics of ticks (Acari: Ixodidae) on horses in the state of São Paulo, Brazil. Vet. Parasitol., v.105, p.65-77, 2002.

LABRUNA, M.B; LEITE, R.C.; GOBESSO, A.A.O. et al. Controle estratégico do carrapato Amblyomma cajennense em eqüinos. Cienc. Rural, v.34, p.195-200, 2004.

LEITE, R.C.; OLIVEIRA, P.R.; LOPES, C.M.L.; FREITAS, C.M.V. Alguns aspectos epidemiológicos das infestações por Amblyomma cajennense: uma proposta de controle estratégico. In: SIMPÓSIO SOBRE CONTROLE DE PARASITOS, 2., 1997, Colina. Anais... Campinas: CGE, 1997. p.9-14. 
LEITE, R.C.; OLIVEIRA, P.R.; LOPES, C.M.L. et al. A febre que vem do carrapato. Vetores $e$ Pragas, v.1, p.22-25, 1998.

LOPES, C.M.L.; LEITE, R.C., LABRUNA, M.B. et al. Host specificity of Amblyomma cajennense (Fabricius, 1787) (Acari: Ixodidae) with comments on the drop-off rhythm. Mem. Inst. Oswaldo Cruz, v.93, p.347-351, 1998.

MILLER, T.A. Mechanisms of resistance to pyrethroids inseticides. Parasitol. Today, v.4, p.8-9, 1988.

OLIVEIRA, P.R. Amblyomma cajennense (Fabricius, 1787) (Acari: Ixodidae) Avaliação de técnica para estudo de dinâmica populacional e bioecologia em Pedro Leopoldo, Minas Gerais, 1998. 110f. Tese (Doutorado) - Escola de Veterinária, Universidade Federal de Minas Gerais, Belo Horizonte.

PINHEIRO, V.R.E. Avaliação do efeito carrapaticida de alguns piretróides sintéticos sobre o carrapato Amblyomma cajennense (Fabricius, 1787) (Acari: Ixodidae). Hora Vet., v.7, p.21-25, 1987.

PRATA, M.C.A.; ALONSO, L.S.; SANAVRIA, A. Parâmetros biológicos do estádio ninfal de Amblyomma cajennense (Fabricius, 1787) (Acari: Ixodidae) em coelhos. Rev. Bras. Cienc. Vet., v.3, p.55-57, 1996.

RAY, D.E. Pesticides derived from plants and other organisms. In: HAYES, W.J.J.; LAWS, E.R.J. (Eds). Handbook of pesticide toxicology: classes of pesticides. California: Academic, 1991. v.2, p.585-636.

RAY, D.E.; FORSHAW, P.J. Pyrethroid insecticides: poisoning syndromes, synergies, and therapy. J. Clin. Toxicol., v.38, p.95-101, 2000.

SAMPAIO, I.B.M. Estatística aplicada à experimentação animal. Belo Horizonte: FEPMVZ, 2002. 265p.
SCHMITZ, D.G. Problemas toxicológicos. In: REED, S.M.; BAYLY, W.M. (Eds). Medicina interna eqüina, Rio de Janeiro: Guanabara Koogan, 2000. p.840-884.

SERRA-FREIRE, N.M. Alterações hematológicas em bovinos leiteiros Holando/zebu induzidas por "carrapato Estrela". Hora Vet., v.4, p.45-48, 1984.

SILVEIRA, J.M. (Ed). Patologia clínica veterinária: teoria e interpretação. Rio de Janeiro: Guanabara, 1988. 196p.

SODERLUND, D.M.; CLARK, J.M.; SHEETS, L.P. et al. Mechanisms of pyrethroid neurotoxicity: implications for cumulative risk assessment. Toxicology, v.171, p.3-59, 2002.

SPEIRS, V.C. (Ed). Exame clínico de eqüinos. Porto Alegre: Artmed, 1999. 366p.

VALENTINE, W.M. Toxicology of selected pesticides, drugs and chemicals: pyrethrin and pyrethroid insecticides. Vet. Clin. N. Am.: Small Anim. Pract., v.20, p.375-403, 1990.

VERÇOSA JÚNIOR, D.; SOUZA-FAGUNDES, E.M.; CASSALI, G.D. et al. Efeito do miriadenolideo isolado de Alomia myriadenia (Asteraceae) sobre o tumor de Ehrlich ascítico no camundongo. Arq. Bras. Med. Vet. Zootec., v.58, p.788-798, 2006.

VIANA, PC.R.; CALDAS-BUSSIERE, M.C.; MARINS, R.S.Q.S. et al. Effect of exercise on occurrence of diurnal rhythms of plasma ions and metabolites in Thoroughbread racehorses. Arq. Bras. Med. Vet. Zootec., v.59, p. 857-861, 2007.

WOLANSKY, M.J.; GENNINGS, C.;

CROFTON, K.M. Relative potencies for acute effects of pyrethroids on motor function in rats. Toxicol. Sci., v.89, p.271-277, 2006. 